\title{
Clinicopathological characteristics of ypTONO urothelial carcinoma following neoadjuvant chemotherapy and cystectomy
}

\author{
Martin J Magers, ${ }^{1}$ Hristos Z Kaimakliotis, ${ }^{2}$ Marcelo P Barboza, ${ }^{2}$ Elhaam Bandali, ${ }^{2}$ \\ Nabil Adra, ${ }^{3}$ Michael O Koch, ${ }^{2}$ Liang Cheng ${ }^{\oplus 1,2}$
}

'Department of Pathology, Indiana University School of Medicine, Indianapolis, Indiana, USA

${ }^{2}$ Department of Urology, Indiana University School of Medicine, Indianapolis, Indiana, USA ${ }^{3}$ Divisoin of Hematology/ Oncology, Indiana University School of Medicine, Indianapolis, Indiana, USA

\section{Correspondence to} Dr Liang Cheng, Department of Pathology \& Laboratory Medicine, Indiana University School of Medicine, Indianapolis, IN 46202, USA liang_cheng@yahoo.com

Received 25 January 2019 Revised 9 May 2019

Accepted 18 May 2019

Published Online First

4 June 2019

\begin{abstract}
Aims To describe a large tertiary care academic centre's experience with patients who achieve a complete pathological response (ie, ypTON0) following neoadjuvant chemotherapy (NAC) and radical cystectomy (RC) with emphasis on morphological features present in the RC and clinical outcome.
\end{abstract}

Methods 41 patients with ypTONO disease following transurethral resection of bladder tumour (TURBT), NAC and $R C$ with available clinical follow-up information were analysed. Slides from all RCs were reviewed to confirm pathological stage and assess for morphological parameters (eg, foreign body giant cell reaction, dystrophic calcification, scar and fat necrosis).

Results With median follow-up of 32.8 months, the recurrence-free survival at 1 and 5 years was $97.4 \%$ and $93.5 \%$, while the overall survival at 3 and 5 years was $94.2 \%$ and $88.6 \%$, respectively. No patients died of urothelial carcinoma. Stage assigned at TURBT was 1 pTa (2\%), 1 pT1 (2\%), 38 pT2 (93\%) and 1 pT3a (2\%). 17 TURBTs demonstrated variant histology, with the majority of these being squamous (65\%). The most common morphological features present at RC were scar $(100 \%)$, foreign body giant cell reaction $(80 \%)$, chronic inflammation within lamina propria (68\%) and dystrophic calcifications (39\%). Other morphological features were less common or absent.

Conclusion ypTONO disease at RC portends an excellent prognosis, regardless of stage or variant histology in the TURBT; scar, foreign body giant cell reaction, chronic inflammation and dystrophic calcifications are often present.

\section{INTRODUCTION}

Urothelial carcinoma (UC) of the urinary bladder is one of the most common types of cancer, with more than 80470 expected new diagnoses and 17670 expected deaths in the USA in $2019 .^{1}$ Clearly, UC of the urinary bladder confers significant mortality, and patients often require an aggressive therapeutic strategy. UC of the urinary bladder is now often treated with radical cystectomy (RC) and lymph node dissection following transurethral resection of bladder tumour (TURBT) and neoadjuvant chemotherapy (NAC). ${ }^{2-5}$ Thus, no residual tumour may be present in the RC specimen (ypT0) or lymph nodes (pNo). ${ }^{6}$

Historically, the incidence of stage ypT0 bladder carcinoma was approximately $10 \% .^{7-12}$ With increased utilisation of NAC, however, the incidence of stage ypT0 bladder carcinoma may be increasing, and recent studies show a stage ypT0 incidence of nearly 30\% following NAC. ${ }^{13-16}$ Indeed, a primary benefit of NAC may be that it increases a patient's chance of achieving stage ypT0, and these patients experience better overall survival (OS) and recurrence-free survival (RFS) than patients without a complete pathological response. ${ }^{13}$ 16-20 Nevertheless, unfavourable outcomes can occur in patients with ypT0, and this is likely due to extravesical spread prior to RC. Lymph node metastases in patients with ypT0 are rare, with $3 \%-7 \%$ of patients developing nodal metastasis. ${ }^{8} 11$

As stage ypT0 bladder carcinoma is more frequently encountered at RC, surgical pathologists and urologists must become comfortable with the absence of residual tumour in the RC and lymph node dissection. ${ }^{21}$ However, detailed description of clinicopathological characteristics of stage ypT0N0 RC specimens following TURBT and NAC is lacking in the literature. Here, we describe a large tertiary care academic centre's experience with patients who achieve a complete pathological response (ie, ypT0N0) following NAC and RC with emphasis on morphological features present in the $\mathrm{RC}$ and clinical outcome.

\section{METHODS}

Patients with stage ypT0N0 disease following TURBT, NAC and RC with available clinical follow-up were identified from the pathology and urology records at Indiana University over a 10-year period (January 2007 to December 2017). All patients underwent cystectomy with curative intent. Urinary diversion technique was decided based on tumour characteristics, surgeon and/or patient preference. An extended template pelvic lymph node dissection (with the bifurcation of the great vessels representing the proximal limit of lymphadenectomy) is the standard dissection at the Indiana University. Patient demographic data (age, gender, and so on) were listed in table 1.

RCs included in the study were grossed by experienced pathologists' assistants (PA) or pathology trainees under the guidance of experienced PAs. In all cases, any grossly suspicious areas of the urinary bladder were sampled. Submission of the entire urinary bladder mucosa was not performed, but in some cases additional sections of the urinary bladder were submitted. Slides from all RCs and lymph node dissections were reviewed to confirm 


\begin{tabular}{|c|c|}
\hline Characteristics & $\%$ (Patients, n) \\
\hline \multicolumn{2}{|l|}{ Gender } \\
\hline Male & $83(34)$ \\
\hline Female & $17(7)$ \\
\hline \multicolumn{2}{|l|}{ Age (years) } \\
\hline Range & $45-78$ \\
\hline Mean & 61 \\
\hline Median & 60 \\
\hline \multicolumn{2}{|l|}{ Stage of TURB } \\
\hline pTa & $2(1)$ \\
\hline pT1 & $2(1)$ \\
\hline$\geq \mathrm{pT} 2$ & $95(39)$ \\
\hline \multicolumn{2}{|l|}{ CIS present } \\
\hline Yes & $29(10)$ \\
\hline No & $71(25)$ \\
\hline \multicolumn{2}{|c|}{ Variant histology in TURB } \\
\hline Squamous & $65(11)^{*}$ \\
\hline Sarcomatoid* & $6(1)^{*}$ \\
\hline Micropapillary & $12(2)$ \\
\hline Plasmacytoid & $6(1)$ \\
\hline Glandular & $6(1)$ \\
\hline Other & $12(2)$ \\
\hline
\end{tabular}

*One patient had both squamous and sarcomatoid differentiation.

CIS, carcinoma in situ; TURBT, transurethral resection of bladder tumour.

pathological stage of ypT0 and nodal stage of pN0. Additionally, the presence or absence of various histological parameters (eg, foreign body giant cell reaction, dystrophic calcification, scar and fat necrosis) was recorded. Surgical pathology reports from prior TURBTs were reviewed.

Patients were subjected to NAC followed by RC at the discretion of a multidisciplinary team of physicians in consultation with each patient based on clinical and pathological information available. Indeed, at Indiana University almost all patients with muscle invasive UC of the urinary bladder are evaluated by medical oncology for consideration of cisplatin-based NAC or enrolment on neoadjuvant therapy clinical trials. Patients who are cisplatin eligible (glomerular filtration rate $>60$, no grade $\geq 2$ neuropathy, no hearing loss, Eastern Cooperative Oncology Group performance status <2, no New York Heart Association class 3 congestive heart failure) are offered neoadjuvant cisplatin-based chemotherapy with dose-dense methotrexate, vinblastine, doxorubicin and cisplatin (MVAC). In all, approximately two-thirds of patients receive NAC at Indiana University.

During follow-up periods, patients were periodically evaluated for evidence of recurrence. Patients were closely followed by a multidisciplinary team with imaging of the upper urinary tract together with abdomen and pelvis (CT or MRI urography), plus chest CT or chest X-ray every 4-6 months in the first 2 years, and then every 6-12 months afterwards. Urine cytology and urethral wash were done every 6-12 months in the first 2 years then as clinically indicated. Recurrence was defined as biopsy proven or having radiographical evidence of local or distant failure. Survival outcomes were calculated from the time of RC to date of death or last follow-up. Time to recurrence was estimated from the time of the cystectomy until the date of recurrence diagnosis by imaging or last follow-up. Additionally, OS and RFS after the cystectomy were appraised using the Kaplan-Meier method. Cancer-specific survival was not assessed since there were no cancer-related deaths in this patient cohort.
Statistical analyses were performed using Stata statistical software release V.15 (StataCorp, College Station, TX, USA).

\section{RESULTS}

Forty-one patients with ypT0N0 who underwent NAC followed by RC were identified from the prospectively maintained pathology and urology databases at Indiana University (table 1). Of these, 34 were male, and seven were female. The mean age at RC was 61 years old (median 60 years, range $45-78$ years). Thirty-one patients received gemcitabine and cisplatin chemotherapy while 10 patients received MVAC. The pathological stage assigned at TURBT was known in all patients, and most $(\mathrm{n}=38,93 \%)$ were $\mathrm{pT} 2$ (ie, invasive into muscularis propria). The remaining patients were $\mathrm{pTa}(\mathrm{n}=1,2 \%$; ie, non-invasive papillary UC), pT1 ( $n=1,2 \%$; ie, invasive into lamina propria) or pT3a $(n=1,2 \%$; ie, invasive into perivesical adipose). Although the true pathological stage is assigned to the RC specimen in accordance with the American Joint Committee on Cancer Staging Manual, Eighth Edition, at our institution a preliminary ' $\mathrm{pT}$ ' stage is assigned to the TURBT specimen to guide patient management. ${ }^{622}$ The presence or absence of urothelial carcinoma in situ (CIS) in the TURBT was known in 35 patients; of these, CIS was present in 10 (29\%) TURBTs. Fifteen patients had a variant histology of UC present in the TURBT. Squamous differentiation was the most common variant histology $(n=11$, $65 \%)$, and one patient had both squamous and sarcomatoid differentiation $(n=1,6 \%)$. Micropapillary $(n=2,12 \%)$, plasmacytoid $(n=1,6 \%)$ and glandular $(n=1,6 \%)$ differentiation were also reported in the TURBTs; 2 (12\%) cases were reported only as 'other'. Muscularis propria was present in all TURBTs. Lymphovascular invasion was present in two TURBT specimens; these patients did not have lymph node metastases identified at the time of RC and lymph node dissection.

The most common histological findings present at cystectomy were scar $(\mathrm{n}=41,100 \%$, table 2, figure 1$)$, foreign body giant cell reaction $(n=33,80 \%)$, chronic inflammation within lamina propria $(n=28,68 \%)$ and dystrophic calcifications $(n=16$, $39 \%)$. Fat necrosis was present in $4(10 \%)$ RCs, including the single patient with invasion into perivesical adipose (stage pT3a) in the previous TURBT. Urothelial dysplasia, defined as atypia

\begin{tabular}{lc}
$\begin{array}{l}\text { Table } 2 \text { Histological parameters observed in radical cystectomy } \\
\text { specimens }\end{array}$ \\
\hline Characteristics & $\%$ (Patients, n) \\
\hline Urothelial dysplasia & $10(4)$ \\
Mucosal ulceration & $17(7)$ \\
Extensively denuding mucosa & $17(7)$ \\
Keratinising squamous metaplasia & $5(2)$ \\
Viral inclusions & $0(0)$ \\
Foreign body giant cell reaction & $80(33)$ \\
Dystrophic calcifications & $39(16)$ \\
Scar & $100(41)$ \\
Fat necrosis & $10(4)$ \\
Mucosa & $17(7)$ \\
Acute inflammation & $15(6)$ \\
Chronic inflammation & $10(4)$ \\
Histiocytic inflammation & $17(7)$ \\
Lamina propria & $15(6)$ \\
Acute inflammation & $10(4)$ \\
\hline Chronic inflammation & \\
Histiocytic inflammation &
\end{tabular}

Magers MJ, et al. J Clin Pathol 2019;72:550-553. doi:10.1136/jclinpath-2019-205742 


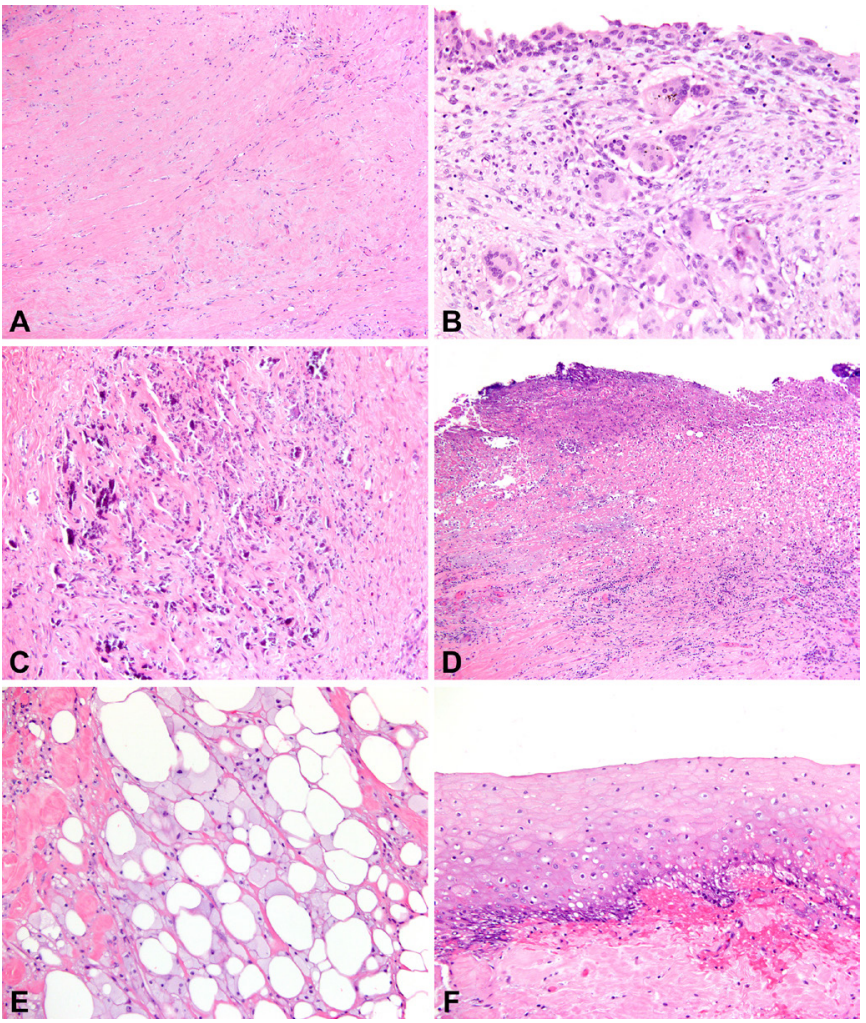

Figure 1 Histopathological appearance of ypTONO bladder cancer. Scar was present in the lamina propria of all radical cystectomy $(R C)$ specimens $(A, H \& E, 100 \times)$. Other histological features which were frequently identified were foreign body giant cell reaction $\left(B, H \& E_{1}\right.$ $200 x$ ), chronic inflammation within the lamina propria and dystrophic calcifications (C, H\&E, 200x). Mucosal ulceration was also sometimes present $(17 \%, D, H \& E, 100 \times)$. Fat necrosis was identified in $4(10 \%)$ $\mathrm{RCs}$, including the single patient with invasion into perivesical adipose diagnosed in the previous transurethral resection of bladder tumour (TURBT) $(E, H \& E, 200 \times)$. Keratinising squamous metaplasia was rarely present $(5 \%, F, H \& E, 200 x)$.

more consistent with neoplasia than reactive changes but without reaching the threshold of CIS, was present in $4(10 \%) \mathrm{RCs}$; two of these patients had CIS present in the previous TURBT while two did not. In addition to chronic inflammation within the lamina propria, acute inflammation $(n=1,2 \%)$ and histiocytic inflammation $(n=4,10 \%)$ were also present in the lamina propria. The surface urothelium was involved by acute inflammation in 7 (17\%) RCs, chronic inflammation in 6 (15\%) RCs and histiocytic inflammation in $4(10 \%)$ RCs. Other histological findings identified in the RCs were mucosal ulceration $(n=7$, $17 \%)$, extensively denuding mucosa $(n=7,17 \%)$ and keratinising squamous metaplasia $(n=2,5 \%)$. Features which were specifically assessed but not identified in any of the RC specimens were cystitis glandularis with intestinal metaplasia $(n=0$, $0 \%)$, viral inclusions $(n=0,0 \%)$, foreign body giant cell reaction associated with cholesterol clefts $(n=0,0 \%)$ and vascular fibroelastotic stroma with macrophages $(n=0,0 \%)$.

In all, three patients died in this cohort. Two died as a result of postoperative complications; one death was due to a complicated intra-abdominal abscess, and the other was due to a pulmonary embolism. The cause of death of the third patient is unknown. Furthermore, during the follow-up period only 2 (4.9\%) patients developed cancer recurrence. One of the recurrences was found on the right adrenal by a positron emission
A

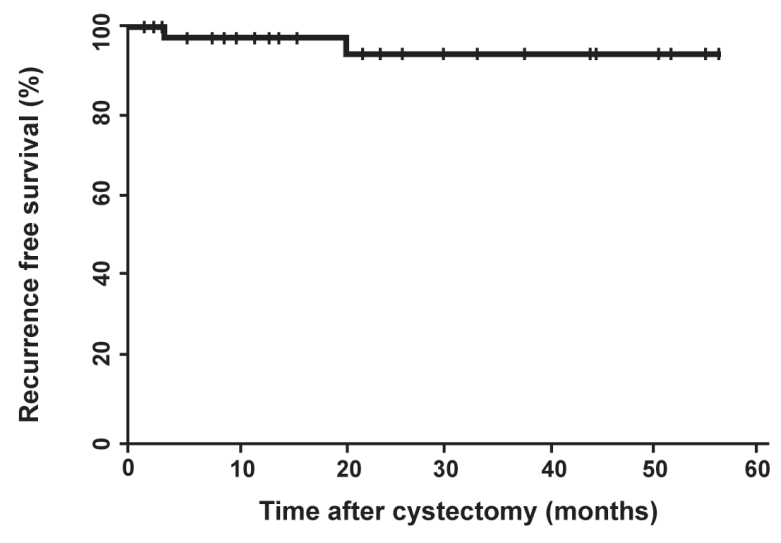

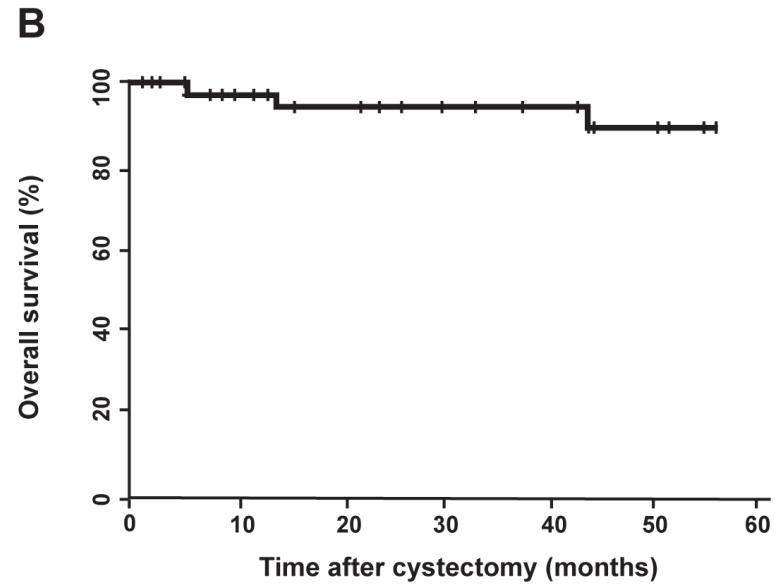

No. at risk: 41 25

18 11

Figure 2 (A) Recurrence-free survival and (B) overall survival for ypTONO bladder cancer.

tomography/CT 19.9 months after the RC and was treated only with adrenalectomy; this patient had no evidence of disease 42.7 months after RC. The second patient was put on atezolizumab for 6 months after a CT showed upper urinary tract recurrence 2.1 months after surgery. She showed no evidence of disease at her last follow-up 14.9 months after RC. The median follow-up after RC was 32.80 (IQR: 13.33, 60.90) months. Furthermore, at 1 and 5 years, the RFS was $97.37 \%$ and $93.47 \%$, while the OS at 3 and 5 years was $94.16 \%$ and $88.62 \%$, respectively. The Kaplan-Meier curves for OS and RFS are shown in figure 2.

\section{DISCUSSION}

In the present study, we reviewed the RC specimen and lymph node dissections of 41 patients with ypT0N0 following NAC and RC. By definition, none of the patients had any residual UC, either in the urinary bladder or in the lymph nodes, and these patients had exceptionally good outcomes, with none of the patients dying from UC. Most of these patients had aggressive, muscle-invasive UC prior to NAC, and a notable subset of them had aggressive morphological variants present in their TURBT (ie, micropapillary, plasmacytoid or sarcomatoid differentiation). Thus, NAC followed by RC appears to be appropriate management for at least a subset of patients with UC. ${ }^{13} 16-20$

As NAC followed by RC increases the proportion of patients who achieve ypT0N0, surgical pathologists will more frequently 
encounter RC and lymph node dissections, which lack any residual UC in patients with a history of aggressive, muscle-invasive UC. Clearly, meticulous gross examination of the surgical resection specimen(s) coupled with histological analysis of any grossly suspicious areas is necessary prior to assigning ypT0N0, given the important prognostic implications. However, it would also be unreasonable to expect histological examination of the entire RC specimen prior to assigning ypT0N0. ${ }^{23}{ }^{24}$ With histological undersampling and oversampling of the urinary bladder at two ends of a spectrum, there is clearly an appropriate midpoint, which involves meticulous gross examination coupled with submission of a reasonable number of blocks for histological analysis. For the surgical pathologist who has yet to routinely encounter ypT0N0 RC specimens, however, this midpoint may be difficult to find.

For this reason, we sought to provide histological parameters, which may be associated with UC effectively treated by NAC. To that end, the most common histological findings of ypT0N0 RC specimens in our study were scar (100\%), foreign body giant cell reaction (80\%) and chronic inflammation in the lamina propria (68\%). These findings are not surprising, but, nonetheless, they support the fact that a RC specimen has been adequately sampled. Importantly, the presence of scar was the only truly ubiquitous histological parameter, and chronic inflammation and a foreign body giant cell reaction were not present in all cases.

In summary, stage ypT0N0 portends an excellent prognosis in patients with UC following NAC and RC. Assignment of ypT0N0 should only occur following a meticulous gross examination of the urinary bladder with histological sampling of any suspicious areas. If a scar is present in the examined sections, the surgical pathologist can be confident that the appropriate area of the urinary bladder was examined. The presence of a foreign body giant cell reaction and/or chronic inflammation in the lamina propria can also further support the notion that a urinary bladder was adequately sampled, but they may not always be present.

\section{Take home messages}

- Urinary bladder cancer is often treated with neoadjuvant chemotherapy and radical cystectomy.

- The incidence of complete pathological response (ypT0) in this setting is increasing.

- Scar and foreign body giant cell reaction are present in cystectomies lacking residual tumour.

- Patients who achieve ypTONO have an excellent prognosis.

\section{Handling editor Cheok Soon Lee.}

Contributors MJM and $L C$ are involved in conception and design of the paper. MJM and LC are responsible for data acquisition, data analysis and writing the article. All the authors (MJM, HZK, MPB, EB, NA, MOK, LC) read, edited and approved the final manuscript.

Funding The authors have not declared a specific grant for this research from any funding agency in the public, commercial or not-for-profit sectors.

Competing interests None declared.

Patient consent for publication Not required.
Ethics approval The study was approved by the Institutional Review Board of Indiana University-Purdue University Indianapolis.

Provenance and peer review Not commissioned; externally peer reviewed.

Data availability statement All data relevant to the study are included in the article or uploaded as supplementary information.

\section{REFERENCES}

1 Siegel RL, Miller KD, Jemal A. Cancer statistics, 2019. CA Cancer J Clin 2019;69:7-34.

2 Massari F, Santoni M, di Nunno V, et al. Adjuvant and neoadjuvant approaches for urothelial cancer: updated indications and controversies. Cancer Treat Rev 2018;68:80-5

3 Witjes JA, Compérat E, Cowan NC, et al. EAU guidelines on muscle-invasive and metastatic bladder cancer: summary of the 2013 guidelines. Eur Urol 2014;65:778-92

4 Grossman HB, Natale RB, Tangen CM, et al. Neoadjuvant chemotherapy plus cystectomy compared with cystectomy alone for locally advanced bladder cancer. $N$ Engl J Med 2003;349:859-66.

5 Advanced Bladder Cancer (ABC) Meta-analysis Collaboration. Neoadjuvant chemotherapy in invasive bladder cancer: update of a systematic review and metaanalysis of individual patient data advanced bladder cancer (ABC) meta-analysis collaboration. Eur Urol 2005:48:202-5.

6 Magers MJ, Lopez-Beltran A, Montironi R, et al. Staging of bladder cancer. Histopathology 2019;74:112-34.

7 Dalbagni G, Genega E, Hashibe M, et al. Cystectomy for bladder cancer: a contemporary series. J Urol 2001;165:1111-6.

8 Palapattu GS, Shariat SF, Karakiewicz PI, et al. Cancer specific outcomes in patients with pT0 disease following radical cystectomy. J Urol 2006;175:1645-9.

9 Kassouf W, Spiess PE, Brown GA, et al. PO stage at radical cystectomy for bladder cancer is associated with improved outcome independent of traditional clinical risk factors. Eur Urol 2007;52:769-76.

10 Thrasher JB, Frazier HA, Robertson JE, et al. Does of stage pT0 cystectomy specimen confer a survival advantage in patients with minimally invasive bladder cancer? J Urol 1994;152:393-6

11 Volkmer BG, Kuefer R, Bartsch G, et al. Effect of a pT0 cystectomy specimen without neoadjuvant therapy on survival. Cancer 2005;104:2384-91.

12 Yiou R, Patard JJ, Benhard H, et al. Outcome of radical cystectomy for bladder cancer according to the disease type at presentation. BJU Int 2002;89:374-8.

13 Petrelli F, Coinu A, Cabiddu M, et al. Correlation of pathologic complete response with survival after neoadjuvant chemotherapy in bladder cancer treated with cystectomy: a meta-analysis. Eur Urol 2014;65:350-7.

14 Zargar H, Espiritu PN, Fairey AS, et al. Multicenter assessment of neoadjuvant chemotherapy for muscle-invasive bladder cancer. Eur Urol 2015;67:241-9.

15 Pokuri VK, Syed JR, Yang Z, et al. Predictors of complete pathologic response (pT0) to neoadjuvant chemotherapy in muscle-invasive bladder carcinoma. Clin Genitourin Cancer 2016;14:e59-65.

16 Sonpavde G, Goldman BH, Speights VO, et al. Quality of pathologic response and surgery correlate with survival for patients with completely resected bladder cancer after neoadjuvant chemotherapy. Cancer 2009;115:4104-9.

17 Lavery HJ, Stensland KD, Niegisch G, et al. Pathological TO following radical cystectomy with or without neoadjuvant chemotherapy: a useful surrogate. J Urol 2014;191:898-906

18 Kim HS, Jeong CW, Kwak C, et al. Pathological T0 following cisplatin-based neoadjuvant chemotherapy for muscle-invasive bladder cancer: a network metaanalysis. Clin Cancer Res 2016;22:1086-94.

19 Brant A, Kates M, Chappidi MR, et al. Pathologic response in patients receiving neoadjuvant chemotherapy for muscle-invasive bladder cancer: is therapeutic effect owing to chemotherapy or TURBT? Urol Oncol 2017;35:34.e17-34.e25.

20 Zargar H, Zargar-Shoshtari K, Lotan Y, et al. Final pathological stage after neoadjuvant chemotherapy and radical cystectomy for bladder Cancer-Does pT0 predict better survival than pTa/Tis/T1? J Urol 2016;195:886-93.

21 Bostwick DG, Ramnani D, Cheng L. Diagnosis and grading of bladder cancer and associated lesions. Urol Clin North Am 1999;26:493-507.

22 Cheng L, Montironi R, Davidson DD, et al. Staging and reporting of urothelia carcinoma of the urinary bladder. Mod Pathol 2009;22(Suppl 2):S70-S95.

23 Cheng L, Lopez-Beltran A, Bostwick DG. Bladder pathology. Hoboken, NJ: WileyBlackwell, 2012.

24 Cheng L, MacLennan GT, Bostwick DG. Urologic surgical pathology. Fourth edition. Philadelphia, PA: Elsevier, 2019. 